\title{
Correction to: Coalescent theories and divergent paraphrases: definites, non-extensional contexts, and familiarity
}

\section{Francesco Pupa ${ }^{1}$ iD}

Published online: 29 March 2021

(C) Springer Nature B.V. 2021

\section{Correction to: Synthese \\ https://doi.org/10.1007/s11229-020-03006-2}

The original article has been updated. Unfortunately, not all corrections were carried out correctly. In several formulae the existential quantifier ' $\exists$ ' was changed to the universal quantifier ' $\forall$ '. This has been corrected in all places.

Affected were the following formulae:

\section{Example 3.}

$\lambda \mathbf{F} . \lambda \mathbf{G} . \exists x[(\mathbf{F x} \& \forall y(\mathbf{F y} \rightarrow x=y)) \& \mathbf{G x}]$.

\section{Example 4.}

$\left(\exists x_{1}\right)\left(\left(\forall x_{2}\right)\left(\mathbf{F} \mathbf{x}_{2} \equiv x_{2}=x_{1}\right) \& \mathbf{G x}_{1}\right)$. (1993: pp. 79-80). (His emphasis).

Example 1d.

$\exists x[(\mathbf{F x} \& \forall y(\mathbf{F y} \rightarrow x=y)) \& \mathbf{G x}]$.

\section{Example 7.}

The original article can be found online at https://doi.org/10.1007/s11229-020-03006-2.

\section{Francesco Pupa}

1 Philosophy Department, Nassau Community College, 1 Education Drive, Garden City, NY 11530, USA 
WONDERS $[S, \exists x(($ GHOST-IN-HAN'S-ATTIC $x \quad \& \quad \forall y$ (GHOST-IN-HAN'SATTIC $y \rightarrow x=y)$ ) \& NOISY $x$ )].

\section{Example 9.}

BELIEVES $[S, \exists x($ GHOST-IN-HAN'S-ATTIC $x)]$.

\section{Example 12.}

$\sim \exists x[($ BOY $x \& \exists y(($ DOG $y \&$ BOUGHT $x, y) \& \forall z(($ DOG $z \&$ BOUGHT $x$, $z) \rightarrow y=z))) \&$ SOLD $x, y]$.

\section{Example 18.}

$\exists x[(($ DOG $x \& x=L A S S I E) \& \forall y(($ DOG $y \& y=L A S S I E) \rightarrow x=y)) \&$ OLD $x]$.

Footnote 36.

$\llbracket \mathbf{n} \boldsymbol{} \rrbracket=\lambda \mathbf{s}_{\mathbf{r}} \cdot \lambda \mathbf{F} . \lambda \mathbf{y}: \exists ! x\left(\mathbf{F x}, s_{r} \& x=y\right) . \imath x\left(\mathbf{F x}, s_{r} \& x=y\right)$ [Where 's $\mathbf{s}_{\mathbf{r}}$ ' denotes a resource situation].

Publisher's Note Springer Nature remains neutral with regard to jurisdictional claims in published maps and institutional affiliations. 